\title{
Exciton Localization and Radiative Lifetimes in CdSe Nanoplatelets
}

\section{Supporting Information}

\author{
David P. Morgan and David F. Kelley* \\ Chemistry and Chemical Biology, University of California Merced, \\ 5200 North Lake Road, Merced, CA 95343
}

$\underline{\text { Section S1: Static and transient absorption spectra }}$
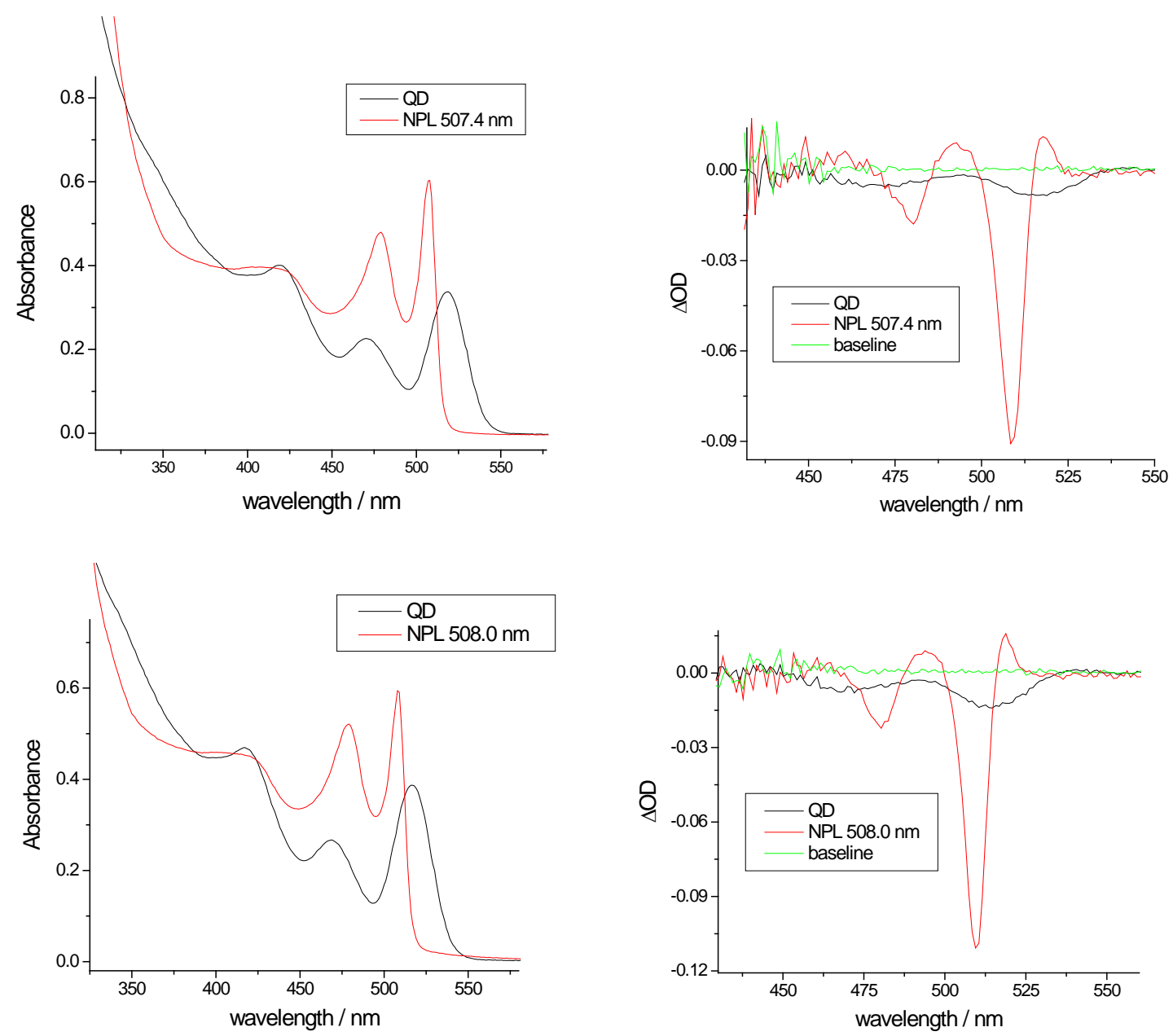

Figure S1. Static absorbance (left) and TA spectra (right) of platelets with areas of 89.5 (top) and 85.5 (bottom) $\mathrm{nm}^{2}$. 

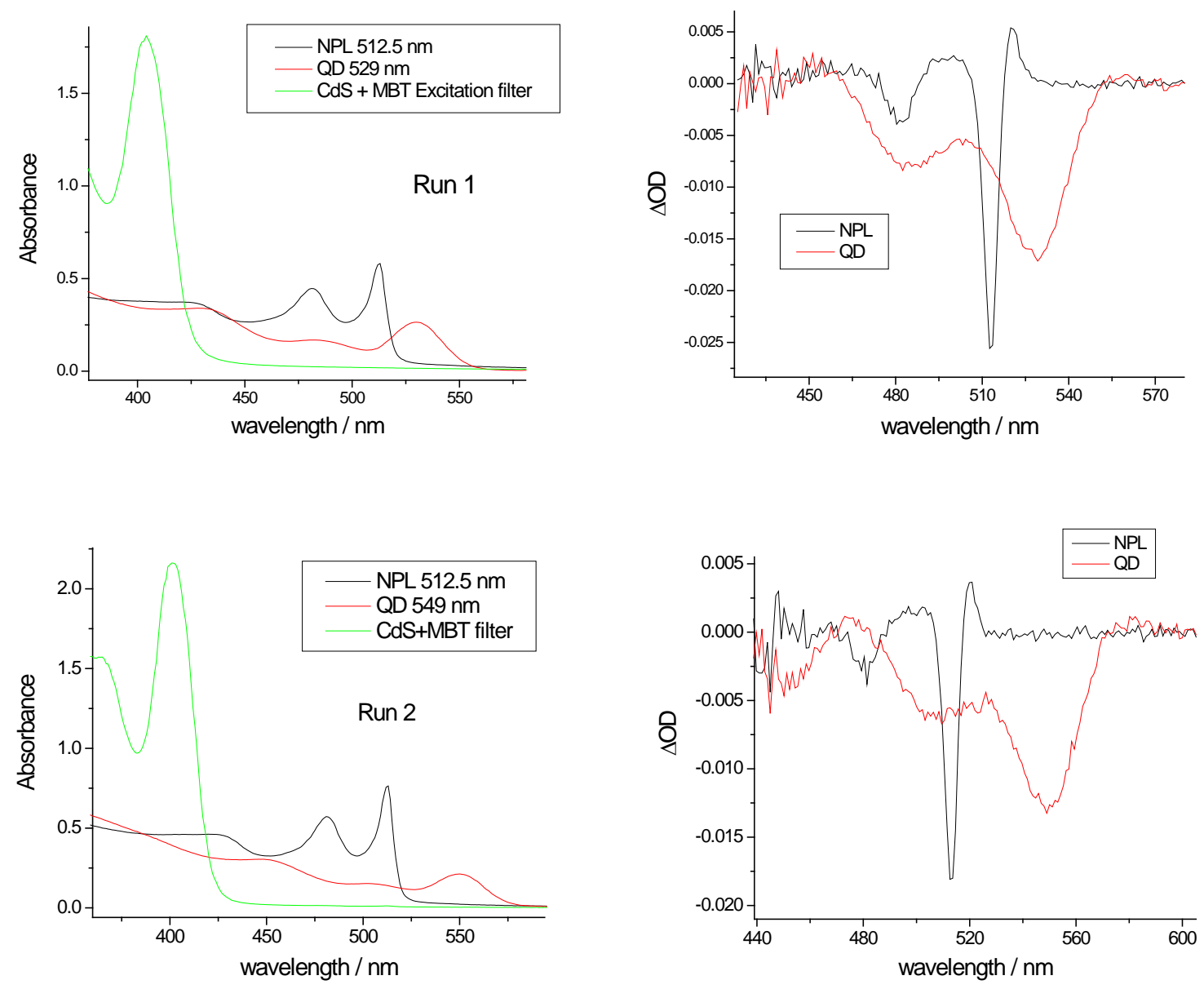

Figure S2. Top left: Static absorbance spectra of the $327 \mathrm{~nm}^{2}$ platelets, QD standard, and excitation filter from Table 1. Top right: TA spectrum of the platelets using the CdS/MBT filter to attenuate the excitation beam coplotted with the QD standard using just hexane as the filter. Bottom: Experiment was repeated using a different QD standard, excitation filter, concentrations, and spectrometer alignment, also in Table 1 . 

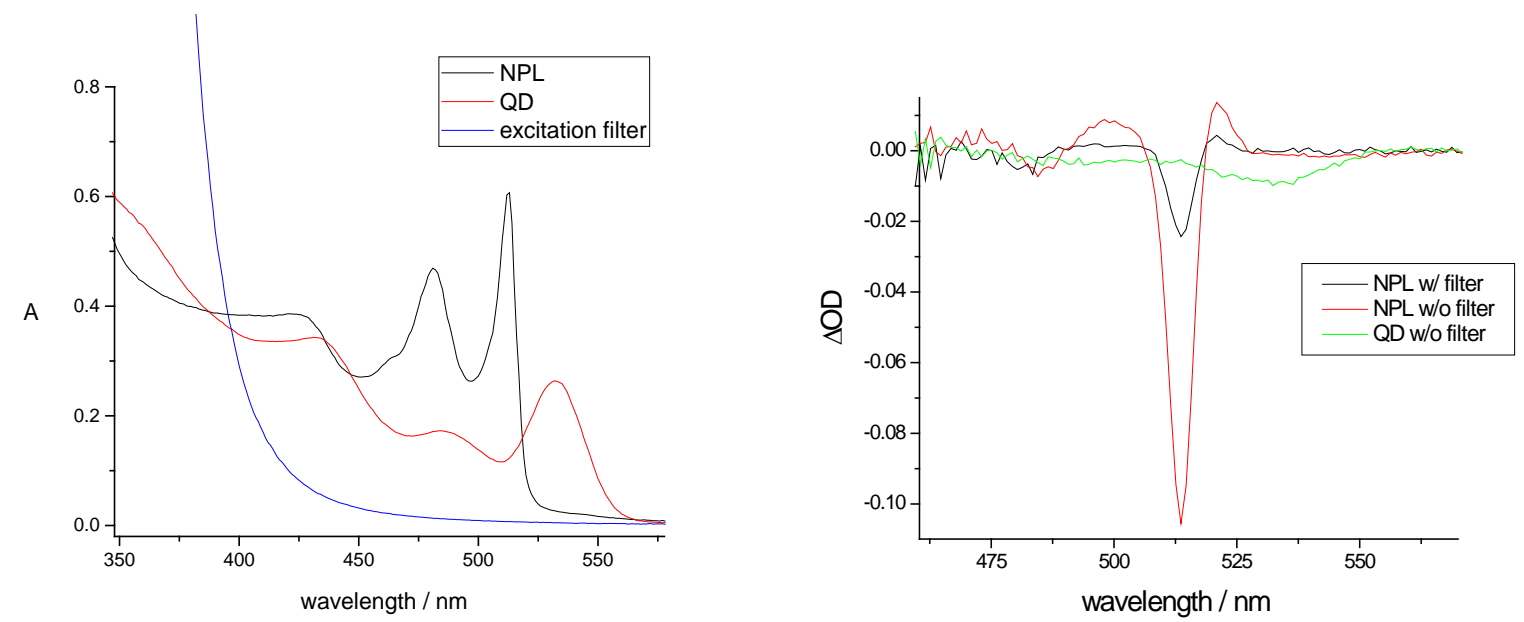

Figure S3. Static absorbance spectra of the $9.3 \times 35.3 \mathrm{~nm}^{2}$ NPL, the QD standard, and a longpass filter used to attenuate the excitation beam. The absorbance of the filter at $387 \mathrm{~nm}$ is 0.67 , indicating that $78 \%$ of the excitation is absorbed. The nanoplatelet bleach magnitudes at the peak of the HH bleach band with and without the excitation filter are 0.024 and 0.103 , constituting a $77 \%$ decrease in the bleach magnitude. We therefore conclude that the NPL bleach magnitudes scale linearly with excitation power in this power regime.
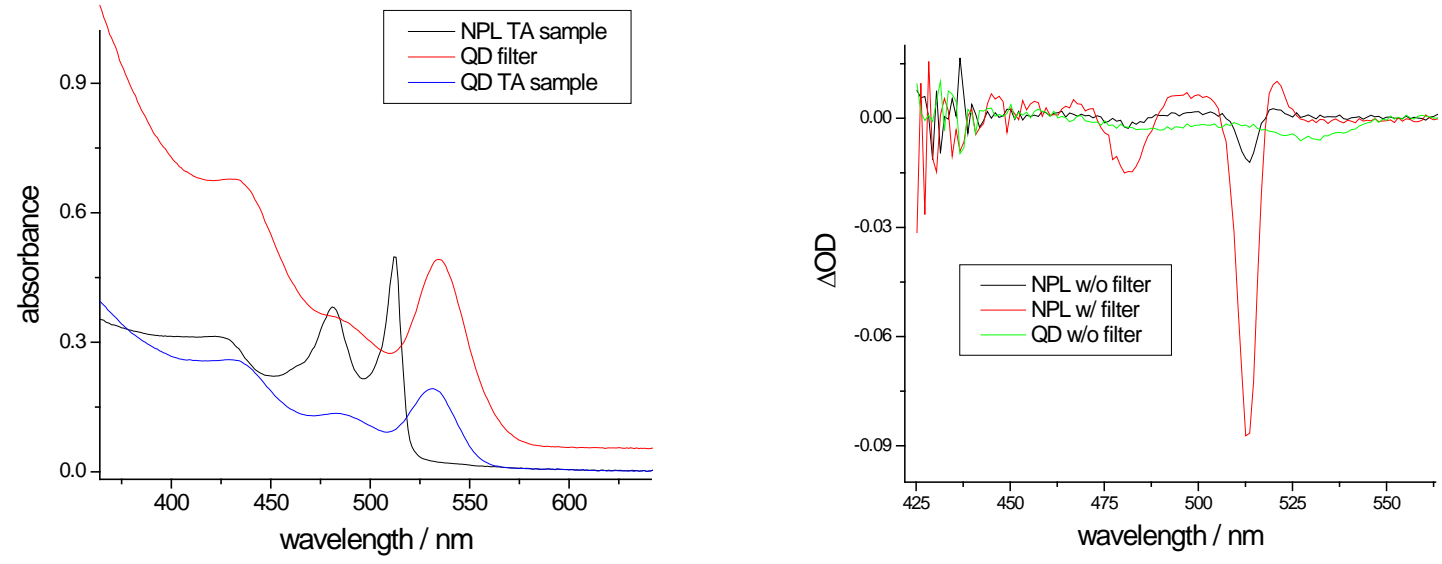

Figure S4. Same as Figure S3, only using a CdSe QD + MBT solution in hexane as a filter to attenuate the excitation beam. The absorbance of the filter at $387 \mathrm{~nm}$ is 0.82 , indicating that $85 \%$ of the excitation is absorbed. The nanoplatelet bleach magnitudes at the peak of the HH bleach band with and without the excitation filter are 0.0118 and 0.0869 , respectively, constituting an $86 \%$ decrease in the bleach magnitude. We therefore conclude that the NPL bleach magnitudes scale linearly with excitation power in this power regime. 
Section S2: TEM images.
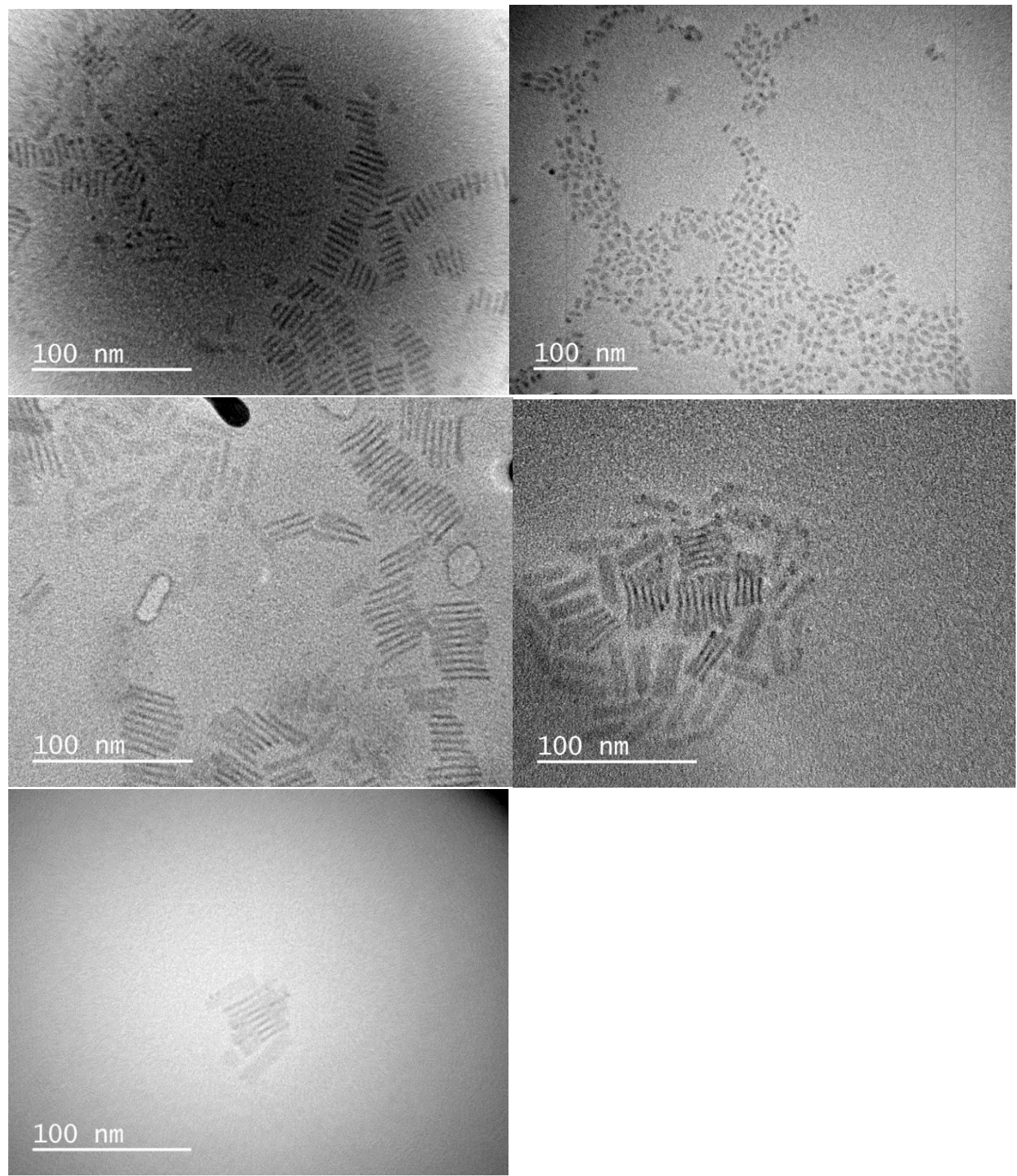

Figure S5: TEM images of each NPL sample presented in Table 1. Top left: $507.4 \mathrm{~nm}, 89.5 \mathrm{~nm}^{2}$. Top right: $508.4 \mathrm{~nm}, 85.5 \mathrm{~nm}^{2}$. Middle left: $509.5 \mathrm{~nm}, 225 \mathrm{~nm}^{2}$. Middle right: $510.8 \mathrm{~nm}, 288 \mathrm{~nm}^{2}$. Bottom left:512.5 nm, $327 \mathrm{~nm}^{2}$. 
Section S3: Simple theoretical model for calculating the spatial extent of a NPL exciton

The magnitude of the $\mathrm{HH}$ bleach band depends on the fraction of platelets that absorb a photon, the size of the exciton relative to that of the platelets, the electron/hole overlap, and the difference in photoinduced absorption and stimulated emission cross-sections of the excitonic state compared to the ground state.

The model considers three distinct regions of the nanoplatelet: the area occupied by the electron and hole $\left(\mathrm{V}_{\mathrm{h}}\right)$, by the electron only $\left(\mathrm{V}_{\mathrm{e}}\right)$, and the region of the platelet not occupied by the exciton. It follows that the total area of the electron (alone and with the hole) is $\mathrm{V}_{\mathrm{x}}=\mathrm{V}_{\mathrm{e}}+\mathrm{V}_{\mathrm{h}}$. The total nanoplatelet area is $\mathrm{V}_{\mathrm{NPL}}$, and the e/h overlap is determined from the change in bleach magnitude upon trapping a hole at MBT.

The situation is simplified by making a few crude approximations:

- The spatial variation of the electron and hole wavefunctions is ignored, with the spatial extents of these wavefunctions characterized only by their areas.

- The spectral shifts of the photoinduced absorption bands are ignored.

- Population of the p-states is ignored.

- After hole trapping, the spatial extent of the electron is unchanged, $\mathrm{V}_{\mathrm{MBT}}=\mathrm{V}_{\mathrm{x}}=\mathrm{V}_{\mathrm{e}}+\mathrm{V}_{\mathrm{h}}$.

\section{Overlap Integral \& Bleach Magnitudes}

The electron and hole wavefunctions are normalized: We take the value of the electron wavefunction to be 1.0 inside the area $V_{x}$ and 0 outside of that area. Normalization requires that $c_{e}^{2} V_{x}=1$. Similarly, we take the value of the hole wavefunction to be 1.0 inside the area $\mathrm{V}_{\mathrm{h}}$ and 0 outside of that area.

Normalization requires that $\mathrm{ch}_{\mathrm{h}}^{2} \mathrm{~V}_{\mathrm{h}}=1$. It follows that the electron-hole overlap integral is

$\mathrm{S}=\mathrm{V}_{\mathrm{h}} \mathrm{C}_{\mathrm{e}} \mathrm{C}_{\mathrm{h}}=\left(\mathrm{V}_{\mathrm{h}} / \mathrm{V}_{\mathrm{x}}\right)^{1 / 2}$

eqn. S1

In this model, the absorbance change is derived as follows: The excited state is given as a product of electron and hole states $|\Psi\rangle=\left|\Psi_{\mathrm{e}}\right\rangle\left|\Psi_{\mathrm{h}}\right\rangle$, each being a constant over areas $\mathrm{V}_{\mathrm{x}}$ and $\mathrm{V}_{\mathrm{h}}$, respectively. The excited state absorbance is obtained by calculating the absorbance at $(\mathrm{x}, \mathrm{y})_{\mathrm{e}}$ and $(\mathrm{x}, \mathrm{y})_{\mathrm{h}}$ and integrating over all $\mathrm{x}, \mathrm{y}$ in $\mathrm{V}_{\mathrm{x}}$ and $\mathrm{V}_{\mathrm{h}}$. When $(\mathrm{x}, \mathrm{y})_{\mathrm{e}}$ are in $\mathrm{V}_{\mathrm{e}}, \mathrm{A}=\mathrm{A}_{0} / 2$. When $(\mathrm{x}, \mathrm{y})_{\mathrm{e}}$ are in $\mathrm{V}_{\mathrm{h}}$, the excited state absorption and stimulated emission have the same magnitude but opposite signs, with the net result being that $\mathrm{A}=0$. We get $\Delta A=A_{x}-A_{0}$, and $\left.A_{x}=(1-f) A_{0}+f\left(\left(1-f_{x}\right) A_{0}+f_{x}\left(c_{e} C_{h}\right)^{2} V_{e} V_{h}\right) A_{0} / 2\right)$, where $f$ is (as before) the fraction of NPLs that absorb a photon and $f_{x}$ is the fraction of the NPL occupied by the exciton, $f_{x}=$ $V_{x} / V_{N P L}$. This gives $A_{x}=(1-f) A_{0}+f\left(\left(1-f_{x}\right) A_{0}+f_{x}\left(V_{e} / V_{x}\right) A_{0} / 2\right)$. It follows that

$-\Delta \mathrm{A} / \mathrm{A}_{0}=\mathrm{ff}_{\mathrm{x}}\left(1-\frac{1}{2}\left(\mathrm{~V}_{\mathrm{e}} / \mathrm{V}_{\mathrm{x}}\right)\right)$

eqn. S2 


\section{Section S4: Derivation of polarization correction to TA spectral intensities}

The axes are defined such that the excitation propagates along the $\mathrm{y}$ axis and is polarized along the $\mathrm{z}$ axis. The probe also propagates along the $y$ axis and is polarized in the $\mathrm{x}-\mathrm{z}$ plane. Photoselection occurs because the probability of absorbing a probe photon depends on the orientation of the pump electric field relative to the NPL transition dipole moment. The NPL absorption at $387 \mathrm{~nm}$ and the lowest exciton are taken to be in-plane polarized. This is a reasonable approximation, although at $387 \mathrm{~nm}$, there may be some out-of-plane component. This analysis treats the QDs as spheres and ignores the differences in local field factors along the nanoplatelet axes, which should be minor approximations.

The distribution of out-of-plane vectors following photon absorption by a planar oscillator is proportional to $\sin ^{2} \theta$, where $\theta$ is the angle of the unique axis with respect to laboratory fixed $\mathrm{z}$ axis. Inclusion of the volume element and normalization gives the distribution over $\theta$ as

$$
P(\theta)=\frac{3}{8 \pi} \sin ^{3}(\theta)
$$

The magnitude of the interaction with a probe photon polarized along $\mathrm{x}$ and $\mathrm{z}$ axes are defined as $\mathrm{I}_{\mathrm{x}}$ and $\mathrm{I}_{\mathrm{z}}$, respectively. These intensities are calculated by taking the projection of the initial distributions of the inplane oscillators along each respective axis, as done in E.S4 and E.S5. To calculate $\mathrm{I}_{\mathrm{x}}$, the integrated projections of the platelet $\mathrm{x}$ and $\mathrm{y}$ axis onto the laboratory frame $\mathrm{x}$ axis are summed. Because $\mathrm{x}$ and $\mathrm{y}$ are equivalent, the sum over all space is 2 , reflecting the two degenerate oscillators. We note that this give the well-known result that the anisotropy is 0.1 .

$$
\begin{array}{ll}
I_{z}=\int_{0}^{2 \pi} d \varphi \int_{0}^{\pi} P(\theta) \sin ^{2} \theta d \theta=2 \pi \frac{3}{8 \pi} \int_{0}^{\pi} \sin ^{5} \theta d \theta=4 / 5 & \text { eqn. S4 } \\
I_{x}=\int_{0}^{2 \pi} \int_{0}^{\pi} P(\theta)\left(\sin ^{2} \varphi \cos ^{2} \theta+\cos ^{2} \varphi\right) d \varphi d \theta=3 / 8 \int_{0}^{\pi} \sin ^{3} \theta d \theta+3 / 8 \int_{0}^{\pi} \sin ^{3} \theta \cos ^{2} \theta d \theta=3 / 5
\end{array}
$$

Probe photons oriented at an angle $\theta$ between $\mathrm{z}$ and $\mathrm{x}$ interact with the distribution of platelet transition dipole moments according to E.S6

$$
I(\theta)=N\left(I_{z} \cos ^{2} \theta+I_{x} \sin ^{2} \theta\right)
$$

Restricting $\theta$ to the interval between 0 and $\pi / 2$ and normalizing gives

$$
I_{N P L}(\theta)=\frac{4}{7 \pi}\left(4 \cos ^{2} \theta+3 \sin ^{2} \theta\right)
$$

QDs are isotropic absorbers, so $\mathrm{I}(\theta)$ is independent of $\theta$. Normalizing this distribution over the same interval gives

$$
I_{Q D}(\theta)=\frac{2}{\pi}
$$


Setting the QD and NPL excited state absorbances equal gives $\theta=\pi / 4$, and this is the angle at which the polarization effect vanishes. However, the experimental geometry uses nearly perpendicular pump and probe orientations, so the nanoplatelet TA spectral intensities are scaled by a factor of $\mathrm{I}_{\mathrm{NPL}}(\pi / 2) / \mathrm{I}_{\mathrm{NPL}}(\pi / 4)$ $=(7 / 2) / 3=1.17$. We find that the bleach ratio at nearly perpendicular $45^{\circ}$ polarization angles is 1.10 . The difference between this value and the calculated value of 1.17 is due to the pump being not entirely orthogonal to the probe pulses and/or some out-of-plane NPL absorption component at the pump wavelength. No further correction due to rotational diffusion is needed because the diffusion times are long compared to the delay times used here. 